Article

\title{
Synthesis and Cytotoxicity Evaluation of Novel Asymmetrical Mono-Carbonyl Analogs of Curcumin (AMACs) against Vero, HeLa, and MCF7 Cell Lines
}

\author{
Pekik Wiji Prasetyaningrum, Anton Bahtiar ${ }^{(D)}$ and Hayun Hayun * (D) \\ Faculty of Pharmacy, Universitas Indonesia, Depok 16424, West Java, Indonesia; pekikwiji@gmail.com (P.W.P.); \\ anton.bahtiar@gmail.com (A.B.) \\ * Correspondence: hayun.ms@ui.ac.id; Tel.: +62-21-727-0031 \\ Received: 8 May 2018; Accepted: 5 June 2018; Published: 7 June 2018

\begin{abstract}
A series of novel asymmetrical mono-carbonyl analogs of curcumin (AMACs) were synthesized and evaluated for cytotoxic activity using BSLT and MTT assay against Vero, HeLa, and MCF7 cell lines. The structures of the synthesized compounds were confirmed by FTIR, ${ }^{1} \mathrm{H}-\mathrm{NMR},{ }^{13} \mathrm{C}-\mathrm{NMR}$, and mass spectral data. The results of the cytotoxicity evaluation showed that the synthesized compounds exhibited moderate to very high toxic activity in BSLT (LC 50 value 29.80-1704.23 $\mu \mathrm{M}$ ); most of the compound exhibited cytotoxic activity against HeLa cell lines, which is comparable to the activity of cisplatin ( $\mathrm{IC}_{50}$ value $40.65-95.55 \mu \mathrm{M}$ ), and most of the compound tested against MCF7 cell lines exhibited moderate to very high cytotoxic activity $\left(\mathrm{IC}_{50}\right.$ value $\left.7.86-35.88 \mu \mathrm{M}\right)$. However, the selectivity index (SI) of the compounds was low $(<1-1.96)$. Among the synthesized compounds, compound $\mathbf{1 b}$ was the most cytotoxic and selective against MCF7 cell lines. It could be considered for further development to obtain the more active and selective chemotherapeutic agents against breast cancer.
\end{abstract}

Keywords: asymmetrical mono-carbonyl analogs of curcumin; AMACs; synthesis; cytotoxicity; Vero; HeLa; MCF7; cell lines

\section{Introduction}

Cancer is one of the main causes of death worldwide, especially in developing countries. Breast and cervix uterine cancer have the highest cancer incidence in Indonesian female populations with 48,998 and 20,928 cases, respectively. The cancer mortality pofile reported in 2014 showed that the mortality caused by the two cancers were $21.4 \%$ and $10.3 \%$ [1]. For many years, chemotherapeutic agents have been developed and used to treat cancer. Unfortunately, there is no drug shows good selectivity for cancer cells. Many chemotherapeutic drugs produce serious chronic and delayed toxicities that may be irreversible, particularly in the heart, lungs, and kidneys [2]. Curcumin (diferuloylmethane) demonstrated various biological activities such as growth suppression in a wide variety of tumor cells, as well as chemopreventive effects on certain types of cancers with low toxicity [3]. Nevertheless, it has not yet been accepted as a therapeutic compound because of its low chemical stability, low solubility, poor absorption, and rapid metabolism, resulting in low bioavailability and weak in vivo biological activity [4-7]. Many curcumin analogs have been synthesized and investigated, such as mono-carbonyl analogs of curcumin (MACs), to improve its bioactivity, stability, and bioavailability.

The mono-carbonyl analogs of curcumin (MACs) exhibited a potency of 10-30 times for cell lines and cellular proteins compared to curcumin [8-11]. Some MACs compounds with acetone and cyclohexanone as a linker between the two phenyl rings inhibited the growth of leukemia, colon, renal, 
melanoma, ovarian, central nervous system (CNS), and prostate cancer cells better than cisplatin [12]. The MACs pharmacokinetic profile is much more stable than curcumin, resulting in higher tumor regression [8,12]. Nowadays, some of the asymmetrical mono-carbonyl analogs of curcumin (AMACs) with different constituents on the two phenyl rings have been developed and reported to show antioxidant, anti-inflammatory, antimicrobial [13-17], and antitumor properties [18]. However, reports on studies of AMACs compounds as anti-cancer agents are still limited. To further explore AMACs as anticancer compounds, we report the synthesis and in vitro cytotoxicity evaluation of novel AMACs (1a-1e and 2a-2e, Scheme 1) against Vero, HeLa, and MCF7 Cell lines.

\section{Materials and Methods}

\subsection{Chemistry}

\subsubsection{General Procedures}

All solvents, chemicals, and reagents were obtained commercially and used without purification. Purity tests of the products were performed using thin layer chromatographic (TLC) method on silica gel 60 F254 plates (Merck, Darmstadt, Germany). Melting points were determined in the capillary tube using melting point apparatus (Stuart Scientific, Bibby Sterilin, Staffordshire, UK) and are uncorrected. Infrared (IR) spectra were recorded on an FTIR $8400 S$ spectrophotometer (Shimadzu, Kyoto, Japan). ${ }^{1} \mathrm{H}-\mathrm{NMR}$ and ${ }^{13} \mathrm{C}-\mathrm{NMR}$ spectra were recorded on Nuclear Magnetic Resonance (NMR) spectrometer (Agilent, Santa Clara, CA, USA) at $500 \mathrm{MHz}$ for $1 \mathrm{H}$ and $125 \mathrm{MHz}$ for ${ }^{13} \mathrm{C}$ using tetramethylsilane (TMS) as internal standard, and High-resolution mass spectra (HRMS) were measured with a Waters LCT Premier XE (ESI-TOF) (Waters Corp., Milford, MA, USA) system in negative mode.

\subsubsection{Synthesis of (2E)-2-(phenylmethylidene)cyclohexan-1-one and Analogs}

The syntheses were performed according to the synthesis method used for 2-benzylidene acetone by replacing acetone with cyclohexanone [19]. A mixture of aromatic aldehyde $(0.32 \mathrm{~mol})$ and cyclohexanone $(0.88 \mathrm{~mol})$ was added to a solution of $\mathrm{NaOH}(10 \%)$ dropwise while stirring for $2 \mathrm{~h}$. The mixture was neutralized with dilute $\mathrm{HCl}$ to $\mathrm{pH} 7$, the organic layer was separated, and the water layer was extracted with $16 \mathrm{~mL}$ of toluene. The toluene layer was mixed with the organic layer, washed with $16 \mathrm{~mL}$ of water, dried with anhydrous sodium sulfate, and evaporated using rotary vacuum evaporator to give the crude product. The crude product was used as the starting material for the next step without further purification.

\subsubsection{Synthesis of Asymmetrical Mono-Carbonyl Analogs of Curcumin (AMACs) (1a-1e)}

The synthesis of the compounds was performed by aldol condensation of (2E)-2-(phenylmethylidene)cyclohexan-1-one or its analogs and vanillin under acidic condition. The mixture of (2E)-2-(phenylmethylidene)cyclohexan-1-one or its analogs $(0.005 \mathrm{~mol})$ and vanillin $(0.01 \mathrm{~mol})$ in ethanol $(10 \mathrm{~mL})$ was heated under reflux condition until dissolved and a drop of diluted $\mathrm{HCl} /$ ethanol (1 drop: $1 \mathrm{~mL}$ ) was added and stirred for 30 mins. The progress of the reaction was monitored using TLC method. Upon completion, the solvent was evaporated, and the solid material obtained was triturated with a cold mixture of glacial acetic acid/water (1:1) and filtered using the Buchner funnel. The solid product obtained was washed with a cold mixture of glacial acetic acid/water (1:1), dried, and purified by column chromatography with a mixture of the appropriate ratio of n-hexane and ethyl acetate.

(2E,6E)-2-[(4-hydroxy-3-methoxyphenyl)methylidene]-6-(phenylmethylidene)cyclohexan-1-one (1a). The compound was a bright yellow powder, in a $50.0 \%$ yield, m.p.: $149-151{ }^{\circ} \mathrm{C}$ and $\mathrm{Rf}=0.8$ (ethyl acetate: n-hexane $=1: 2)$. FTIR $(\mathrm{KBr})$ vmax cm $\mathrm{cm}^{-1}: 3211(\mathrm{OH}), 2999(\mathrm{CH}$ aromatic), 2837 (CH aliphatic), 1647 (C=O), 1587, 1531, 1448 (C=C), 1174 (C-O). ${ }^{1} \mathrm{H}-\mathrm{NMR}\left(500 \mathrm{MHz}, \mathrm{CDCl}_{3}\right.$ ), 8/ppm: 1.80 (m, 2H, 
C- $\left.-\mathrm{CH}_{2}-\mathrm{C}_{\text {cyclohexanone }}\right) ; 2.93\left(\mathrm{~m}, 4 \mathrm{H},=\mathrm{C}-\mathrm{CH}_{2}-\mathrm{C}_{\text {cyclohexanone }}\right) ; 3.91\left(\mathrm{~s}, 3 \mathrm{H}, \mathrm{OCH}_{3}\right) ; 5.89(\mathrm{~s}, 1 \mathrm{H}, \mathrm{OH}) ; 6.95$ $\left(\mathrm{d}, 1 \mathrm{H}, J=8.5, \mathrm{H}_{\mathrm{Ar}}\right) ; 7.00\left(\mathrm{~s}, 1 \mathrm{H}, \mathrm{H}_{\mathrm{Ar}} ; 7.09\left(\mathrm{~d}, 1 \mathrm{H}, J=8.3 \mathrm{~Hz}, \mathrm{H}_{\mathrm{Ar}}\right) ; 7.33\left(\mathrm{t}, 1 \mathrm{H}, J=7.4, \mathrm{H}_{\mathrm{Ar}}\right) ; 7.39(\mathrm{t}\right.$, $\left.2 \mathrm{H}, J=7.3 \mathrm{~Hz}, \mathrm{H}_{\mathrm{Ar}}\right) ; 7.46\left(\mathrm{~d}, 2 \mathrm{H}, J=7.2 \mathrm{~Hz}, \mathrm{H}_{\mathrm{Ar}}\right) ; 7.75$ and $7.79(\mathrm{~s}, 1 \mathrm{H} \mathrm{Ar}-\mathrm{CH}=\mathrm{C}$ and $1 \mathrm{H}, \mathrm{C}=\mathrm{CH}-\mathrm{Ar})$. ${ }^{13} \mathrm{C}-\mathrm{NMR}\left(100 \mathrm{MHz}, \mathrm{CDCl}_{3}\right) \delta / \mathrm{ppm}: 23.1$ (1C, C- $\left.\underline{\mathrm{H}}_{2}-\mathrm{C}_{\text {cyclohexanone }}\right), 28.5\left(1 \mathrm{C},=\mathrm{C}-\underline{C H}_{2}-\mathrm{C}_{\text {cyclohexanone }}\right)$, $28.7\left(1 \mathrm{C},=\mathrm{C}-\underline{\mathrm{CH}}_{2}-\mathrm{C}_{\text {cyclohexanone }}\right), 56.0\left(\mathrm{O}-\underline{\mathrm{CH}}_{3}\right), 113.4,114.5,124.6,128.6,130.4,134.2\left(10 \mathrm{C}, \mathrm{C}_{\mathrm{Ar}}\right), 136.1$, 137.5 (4C, $-\mathrm{C}=\mathrm{C}-) 146.4\left(2 \mathrm{C}, \mathrm{C}_{\mathrm{Ar}}-\mathrm{O}\right), 190.3(1 \mathrm{C}, \mathrm{C}=\mathrm{O})$ [20]. HRESIMS $(\mathrm{m} / z)$ found $319.1346\left([\mathrm{M}-\mathrm{H}]^{-}\right)$ calculated masses for $\mathrm{C}_{21} \mathrm{H}_{19} \mathrm{O}_{3}: 319.1334$.

(2E,6E)-2-[(4-hydroxy-3-methoxyphenyl)methylidene]-6-[(4-methoxyphenyl)methylidene] cyclohexan-1-one (1b). The compound was a yellow powder, in a $2.7 \%$ yield, m.p.: $133-136{ }^{\circ} \mathrm{C}$ and $\mathrm{Rf}=0.55$ (ethyl acetate: $n$-hexane = 1:2). FTIR $(\mathrm{KBr})$ vmax $\mathrm{cm}^{-1}: 3431(\mathrm{OH}), 3003(\mathrm{CH}$ aromatic), $2935(\mathrm{CH}$ aliphatic), $1734(\mathrm{C}=\mathrm{O})$, 1656, 1593 and $1512(\mathrm{C}=\mathrm{C}), 1161(\mathrm{C}-\mathrm{O}) .{ }^{1} \mathrm{H} \mathrm{NMR}\left(500 \mathrm{MHz}, \mathrm{CDCl}_{3}\right), \delta / \mathrm{ppm}: 1.80(\mathrm{~m}, 2 \mathrm{H}$, C- $\left.\mathrm{CH}_{2}-\mathrm{C}_{\text {cyclohexanone }}\right) ; 2.92\left(\mathrm{~m}, 4 \mathrm{H},=\mathrm{C}-\mathrm{CH}_{2}-\mathrm{C}_{\text {cyclohexanone }}\right) ; 3.84$ (s, 3H, $\left.\mathrm{CH}_{3}-\mathrm{O}\right) ; 3,91\left(\mathrm{~s}, 3 \mathrm{H}, \mathrm{CH}_{3}-\mathrm{O}\right)$; $5.86(\mathrm{~s}, 1 \mathrm{H}, \mathrm{OH}) ; 6.92\left(\mathrm{~d}, 1 \mathrm{H}, J=8.5, \mathrm{H}_{\mathrm{Ar}}\right) ; 6.99\left(\mathrm{~s}, 1 \mathrm{H}, \mathrm{H}_{\mathrm{Ar}}\right) ; 7.08\left(\mathrm{~d}, 1 \mathrm{H}, J=8.3 \mathrm{~Hz}, \mathrm{H}_{\mathrm{Ar}}\right) ; 7.46(\mathrm{~d}$, $\left.2 \mathrm{H}, J=8.3, \mathrm{~Hz}, \mathrm{H}_{\mathrm{Ar}}\right) ; 7.73$ and $7.76(\mathrm{~s}, 1 \mathrm{H}, \mathrm{Ar}-\mathrm{CH}=\mathrm{C}$ and $\mathrm{s}, 1 \mathrm{H}, \mathrm{C}=\mathrm{CH}-\mathrm{Ar}) .{ }^{13} \mathrm{C}-\mathrm{NMR}(100 \mathrm{MHz}$, $\left.\mathrm{CDCl}_{3}\right) \delta /$ ppm: 23.1 (1C, C- $\left.\underline{\mathrm{CH}}_{2}-\mathrm{C}_{\text {cyclohexanone }}\right), 28.5$ (2C, =C- $\left.\underline{\mathrm{CH}}_{2}-\mathrm{C}_{\text {cyclohexanone }}\right), 56.0\left(1 \mathrm{C}, \mathrm{OCH}_{3}\right)$, $55.4\left(1 \mathrm{C}, \mathrm{OCH}_{3}\right), 113.3,114.4,124.5,128.5,132.3\left(9 \mathrm{C}, \mathrm{C}_{\mathrm{Ar}}\right), 134.4,137.0(4 \mathrm{C}, \mathrm{C}=\mathrm{C}-), 146.4,146.5,160.0$ $\left(3 \mathrm{C}, \mathrm{C}_{\mathrm{Ar}}-\mathrm{O}\right) 190.1(1 \mathrm{C}, \mathrm{C}=\mathrm{O})$ [20]. HRESIMS $(\mathrm{m} / z)$ found $349.1432\left([\mathrm{M}-\mathrm{H}]^{-}\right)$calculated masses for $\mathrm{C}_{22} \mathrm{H}_{21} \mathrm{O}_{4}: 349.1440$.

(2E,6E)-2-[(4-hydroxy-3-methoxyphenyl)methylidene]-6-[(4-fluorophenyl)methylidene] cyclohexan-1-one (1c). The compound was a light yellow powder, in a $47.3 \%$ yield, m.p.: $129-131{ }^{\circ} \mathrm{C}$ and $\mathrm{Rf}=0.52$ (ethyl acetate : n-hexane =1:2). FTIR (KBr) vmax cm $\mathrm{cm}^{-1}: 3313(\mathrm{OH}), 3003(\mathrm{CH}$ aromatic), 2939 (CH aliphatic), $1734(\mathrm{C}=\mathrm{O})$, 1656, 1604 and $1514(\mathrm{C}=\mathrm{C}), 1220(\mathrm{C}-\mathrm{F}), 1155(\mathrm{C}-\mathrm{O}) .{ }^{1} \mathrm{H}-\mathrm{NMR}\left(500 \mathrm{MHz}, \mathrm{CDCl}_{3}\right), \delta / \mathrm{ppm}$ : $1.82\left(\mathrm{~m}, 2 \mathrm{H}, \mathrm{C}-\mathrm{CH}_{2}-\mathrm{C}_{\text {cyclohexanone }}\right) ; 2.89\left(\mathrm{t}, 2 \mathrm{H}, \mathrm{J}=7.1 \mathrm{~Hz},=\mathrm{C}-\mathrm{CH}_{2}-\mathrm{C}_{\text {cyclohexanone }}\right) ; 2.93(\mathrm{t}, 2 \mathrm{H}, \mathrm{J}=6.1 \mathrm{~Hz}$, $\left.=\mathrm{C}-\mathrm{CH}_{2}-\mathrm{C}_{\text {cyclohexanone }}\right) ; 3.93\left(\mathrm{~s}, 3 \mathrm{H}, \mathrm{CH}_{3}-\mathrm{O}\right) ; 5.90(\mathrm{~s}, 1 \mathrm{H},-\mathrm{OH}) ; 6.96\left(\mathrm{~d}, 1 \mathrm{H}, J=9.3 \mathrm{~Hz}, \mathrm{H}_{\mathrm{Ar}}\right) ; 7.00(\mathrm{~s}, 1 \mathrm{H}$, $\left.\mathrm{H}_{\mathrm{Ar}}\right) ; 7.11\left(\mathrm{~d}, 3 \mathrm{H}, J=8.2 \mathrm{~Hz}, \mathrm{H}_{\mathrm{Ar}}\right) ; 7.44\left(\mathrm{dd}, 2 \mathrm{H}, J=5.5 \mathrm{~Hz}, \mathrm{H}_{\mathrm{Ar}}\right) ; 7.75(\mathrm{~s}, 2 \mathrm{H}, \mathrm{Ar}-\mathrm{CH}=\mathrm{C}) .{ }^{13} \mathrm{C} \mathrm{NMR}$ (100 MHz, $\left.\mathrm{CDCl}_{3}\right) \delta /$ ppm: 23.1 (1C, C- $\left.\underline{\mathrm{CH}}_{2}-\mathrm{C}_{\text {cyclohexanone }}\right), 28.7$ (2C, =C- $\left.\underline{\mathrm{CH}}_{2}-\mathrm{C}_{\text {cyclohexanone }}\right), 56.0$ (1C, $\left.\mathrm{OCH}_{3}\right), 113.4,114.5,115.5,115.6,124.6,128.5,132.3,134.1(9 \mathrm{C}, \mathrm{C}$ Ar $), 135.5,136.0,137.8,146.4(4 \mathrm{C}, \mathrm{C}=\mathrm{C})$, $163.7\left(\mathrm{C}_{\mathrm{Ar}}-\mathrm{F}\right), 146.7,160.7\left(2 \mathrm{C}, \mathrm{C}_{\mathrm{Ar}}-\mathrm{O}\right), 190.1(1 \mathrm{C}, \mathrm{C}=\mathrm{O})$ [20]. HRESIMS $(\mathrm{m} / \mathrm{z})$ found $337.1270([\mathrm{M}-$ $\mathrm{H}]^{-}$) calculated masses for $\mathrm{C}_{21} \mathrm{H}_{18} \mathrm{FO}_{3}: 337.1240$.

(2E,6E)-2-[(4-hydroxy-3-methoxyphenyl)methylidene]-6-[(4-chlorophenyl)methylidene] cyclohexan-1-one (1d). The compound was a light yellow powder, in a $6.1 \%$ yield, m.p.: $150-154{ }^{\circ} \mathrm{C}$ and $\mathrm{Rf}=0.75$ (ethyl acetate:n-hexane = 1:2). FTIR (KBr) vmax cm $\mathrm{cm}^{-1}: 3296(\mathrm{OH}), 3003(\mathrm{CH}$ aromatic), 2939 (CH aliphatic), $1734(\mathrm{C}=\mathrm{O})$, 1658, 1604 and $1514(\mathrm{C}=\mathrm{C}), 1163(\mathrm{C}-\mathrm{O}), 833(\mathrm{C}-\mathrm{Cl}) .{ }^{1} \mathrm{H}-\mathrm{NMR}\left(500 \mathrm{MHz}, \mathrm{CDCl}_{3}\right), \delta / \mathrm{ppm}$ : $1.80\left(\mathrm{~m}, 2 \mathrm{H}, \mathrm{C}-\mathrm{CH}_{2}-\mathrm{C}_{\text {cyclohexanone }}\right) ; 2.87\left(\mathrm{t}, 2 \mathrm{H}, \mathrm{J}=7.3 \mathrm{~Hz},=\mathrm{C}-\mathrm{CH}_{2}-\mathrm{C}_{\text {cyclohexanone }}\right) ; 2.93(\mathrm{t}, 2 \mathrm{H}, \mathrm{J}=7.3 \mathrm{~Hz}$, $\left.=\mathrm{C}-\mathrm{CH}_{2}-\mathrm{C}_{\text {cyclohexanone }}\right) ; 3.92\left(\mathrm{~s}, 3 \mathrm{H}, \mathrm{CH}_{3}-\mathrm{O}\right) ; 5.86(\mathrm{~s}, 1 \mathrm{H}, \mathrm{OH}) ; 6.96\left(\mathrm{~d}, 1 \mathrm{H}, J=82 \mathrm{~Hz}, \mathrm{H}_{\mathrm{Ar}}\right) ; 6.99$ (s, $\left.1 \mathrm{H}, \mathrm{H}_{\mathrm{Ar}}\right) ; 7.08\left(\mathrm{~d}, 1 \mathrm{H}, J=8.2 \mathrm{~Hz}, \mathrm{H}_{\mathrm{Ar}}\right) ; 7.37\left(\mathrm{~d}, 2 \mathrm{H}, J=8.8 \mathrm{~Hz}, \mathrm{H}_{\mathrm{Ar}}\right) ; 7,39\left(\mathrm{~d}, 2 \mathrm{H}, J=9.3 \mathrm{~Hz}, \mathrm{H}_{\mathrm{Ar}}\right)$, 7.72, 7.74 (s, 1H, Ar-CH=C and s, 1H, C=CH-Ar). ${ }^{13} \mathrm{C}-\mathrm{NMR}\left(100 \mathrm{MHz}, \mathrm{CDCl}_{3}\right) \delta / \mathrm{ppm}: 23.0(1 \mathrm{C}$, C- $\left.\underline{C H}_{2}-\mathrm{C}_{\text {cyclohexanone }}\right), 28.5,28.6\left(2 \mathrm{C},=\mathrm{C}-\underline{\mathrm{CH}}_{2}-\mathrm{C}_{\text {cyclohexanone }}\right), 56.1\left(1 \mathrm{C}, \mathrm{OCH}_{3}\right), 113.4,114.5,124.7,128.5$ (C15), 129.2, 131.6., 134.1 (9C, $\left.\mathrm{C}_{\mathrm{Ar}}\right), 134.6,135.2,136.8,137.8$ (4C, $\left.-\mathrm{C}=\mathrm{C}-\right)$, 134.0. $\left(\mathrm{C}_{\mathrm{Ar}}-\mathrm{Cl}\right), 146.6,146.7$ (2C, $\left.\mathrm{C}_{\mathrm{Ar}}-\mathrm{O}\right), 196.1(1 \mathrm{C}, \mathrm{C}=\mathrm{O})$ [20]. HRESIMS (m/z) found $353.0947\left([\mathrm{M}-\mathrm{H}]^{-}\right)$calculated masses for $\mathrm{C}_{21} \mathrm{H}_{18} \mathrm{ClO}_{3}: 353.0945$.

(2E,6E)-2-[(4-hydroxy-3-methoxyphenyl)methylidene]-6-[(4-methylphenyl)methylidene] cyclohexan-1-one (1e). The compound was a yellow powder, in a $13.2 \%$ yield, m.p.: $130-131{ }^{\circ} \mathrm{C}$ and $\mathrm{Rf}=0.75$ (ethyl acetate : n-hexane = 1:2). FTIR (KBr) vmax cm $\mathrm{cm}^{-1}: 3323(\mathrm{OH}), 3007$ (CH aromatic), 2939 (CH aliphatic), 1734 $(\mathrm{C}=\mathrm{O}), 1653,1593$ and 1462 (C=C), 1161 (C-O). ${ }^{1} \mathrm{H}-\mathrm{NMR}\left(500 \mathrm{MHz}, \mathrm{CDCl}_{3}\right), \delta / \mathrm{ppm}: 1.79$ (m, 2H, $\left.\mathrm{C}-\mathrm{CH}_{2}-\mathrm{C}_{\text {cyclohexanone }}\right) ; 2.38\left(\mathrm{~s}, 3 \mathrm{H}, \mathrm{CH}_{3}-\mathrm{Ar}\right), 2.91\left(\mathrm{t}, 2 \mathrm{H}, \mathrm{J}=5.0 \mathrm{~Hz},=\mathrm{C}-\mathrm{CH}_{2}-\mathrm{C}_{\text {cyclohexanone }}\right) ; 2.93(\mathrm{t}, 2 \mathrm{H}$, 
$\left.J=5.0 \mathrm{~Hz},=\mathrm{C}-\mathrm{CH}_{2}-\mathrm{C}_{\text {cyclohexanone }}\right), 3.92\left(\mathrm{~s}, 3 \mathrm{H}, \mathrm{CH}_{3}-\mathrm{O}\right) ; 5.86(\mathrm{~s}, 1 \mathrm{H},-\mathrm{OH}) ; 6.96\left(\mathrm{t}, 3 \mathrm{H}, J=8.2 \mathrm{~Hz}, \mathrm{H}_{\mathrm{Ar}}\right)$; $\left.6.99\left(\mathrm{~s}, 1 \mathrm{H}, \mathrm{H}_{\mathrm{Ar}}\right) ; 7.08(\mathrm{~d}, 1 \mathrm{H}, J=8.3 \mathrm{~Hz}, \mathrm{C} 15=\mathrm{CH}-\mathrm{C} 18)\right) ; 7.22\left(\mathrm{~d}, 2 \mathrm{H}, J=9.0 \mathrm{~Hz}, \mathrm{H}_{\mathrm{Ar}}\right) ; 7.37(\mathrm{~d}, 2 \mathrm{H}, J=9.0$ $\left.\mathrm{Hz}, \mathrm{H}_{\mathrm{Ar}}\right), 7,74$ and $7.77(\mathrm{~s}, 1 \mathrm{H}, \mathrm{s}, 1 \mathrm{H}, \mathrm{Ar}-\mathrm{CH}=\mathrm{C}$ and s, $1 \mathrm{H}, \mathrm{C}=\mathrm{CH}-\mathrm{Ar}) .{ }^{13} \mathrm{C}-\mathrm{NMR}\left(100 \mathrm{MHz}, \mathrm{CDCl}_{3}\right)$

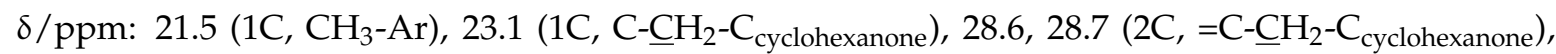
$56.0\left(\mathrm{CH}_{3}-\mathrm{O}\right), 113.4,114.5,124.6,128.6,129.2,130.5,133.3,134.3\left(10 \mathrm{C}, \mathrm{C}_{\mathrm{Ar}}\right), 134.6,135.2,136.8,137.8$ $(4 \mathrm{C},-\mathrm{C}=\mathrm{C}-), 146.4,146.5\left(2 \mathrm{C}, \mathrm{C}_{\mathrm{Ar}}-\mathrm{O}\right), 190.3(1 \mathrm{C}, \mathrm{C}=\mathrm{O})$ [20]. HRESIMS $(\mathrm{m} / \mathrm{z})$ found $333.1492\left([\mathrm{M}-\mathrm{H}]^{-}\right)$ calculated masses for $\mathrm{C}_{22} \mathrm{H}_{21} \mathrm{O}_{3}: 333.1491$.

\subsubsection{Synthesis of Diethylamine Mannich Base of AMACs (2a-2e)}

The syntheses were performed according to the method used for the synthesis of di-Mannich bases of curcumin and the synthesis of 2-[(2,6-dimethylmorpholin-4-yl)methyl]-4-[(E)-2-\{3[(E)-2-\{3[(2,6-dimethylmorpholin-4-yl)methyl]-4-hydroxy-5-methoxyphenyl\}ethenyl]-1H-pyrazol-5yl\}etheyl]-6methoxyphenol reported previously [21,22]. Compound 1a-1e (2 mmol) separately were dissolved in ethanol, cooled in an ice bath, and diethylamine $(5-7 \mathrm{mmol})$ and formaldehyde solution $37 \%$ (5-7 mmol) were added slowly. The mixture was stirred for $30 \mathrm{~min}$ at room temperature and then refluxed for $7-11 \mathrm{~h}$. The progress of the reaction was monitored by TLC. After the reaction was completed, the solvent was evaporated to obtain the solid residue. The residue was dissolved in methanol $(40 \mathrm{~mL})$ and evaporated to a residue. The residue was then dissolved in methanol $(50 \mathrm{~mL})$, warmed, and poured slowly with constant stirring into about $400 \mathrm{~mL}$ of cold distilled water. The solvent was decanted, and the precipitate obtained was filtered off, washed with cold distilled water, dried at room temperature, and then purified by column chromatography.

(2E,6E)-2-(\{3-[(diethylamino)methyl]-4-hydroxy-5-methoxyphenyl\}methylidene)-6-(phenylmethylidene)cyclohexan1-one (2a). The compound was a caramel-like solid, in a $65.5 \%$ yield, m.p.: $79-80{ }^{\circ} \mathrm{C}$ and $\mathrm{Rf}=0.51$ (ethyl acetate: ethanol =1:1). FTIR $(\mathrm{KBr})$ vmax $\mathrm{cm}^{-1}$ : $3053(\mathrm{CH}$ aromatic), $2972(\mathrm{C}-\mathrm{H}), 1660(\mathrm{C}=\mathrm{O})$, 1599 (C=C), 1269 (C-N), 1157 (C-O). ${ }^{1} \mathrm{H}-\mathrm{NMR}\left(500 \mathrm{MHz}, \mathrm{CD}_{3} \mathrm{OD}\right), \delta / \mathrm{ppm}: 1.18$ (t, 6H, J = 7.2 Hz, $\left.\mathrm{CH}_{3}-\mathrm{CH}_{2}-\right), 1.77\left(\mathrm{~m}, 2 \mathrm{H}, \mathrm{C}-\mathrm{CH}_{2}-\mathrm{C}_{\text {cyclohexanone }}\right) ; 2.77\left(\mathrm{q}, 4 \mathrm{H}, \mathrm{J}=7.0 \mathrm{~Hz}, \mathrm{CH}_{3}-\mathrm{CH}_{2}-\mathrm{N}\right), 2.89$ and $2.94(\mathrm{t}$, $\left.4 \mathrm{H}, J=7.0 \mathrm{~Hz},=\mathrm{C}-\mathrm{CH}_{2}-\mathrm{C}_{\text {cyclohexanone }}\right), 3.85\left(\mathrm{~s}, 3 \mathrm{H}, \mathrm{CH}_{3}-\mathrm{O}\right), 3.95\left(\mathrm{~s}, 2 \mathrm{H}, \mathrm{Ar}_{-} \mathrm{CH}_{2}-\mathrm{N}\right), 6.92\left(\mathrm{~s}, 1 \mathrm{H}, \mathrm{H}_{\mathrm{Ar}}\right)$, $7.04\left(\mathrm{~s}, 1 \mathrm{H}, \mathrm{H}_{\mathrm{Ar}}\right) ; 7.33\left(\mathrm{t}, 1 \mathrm{H}, J=8,7 \mathrm{~Hz}, \mathrm{H}_{\mathrm{Ar}}\right) ; 7.40\left(\mathrm{t}, 2 \mathrm{H}, J=7.4 \mathrm{~Hz} \mathrm{H}_{\mathrm{Ar}}\right), 7.45(\mathrm{~d}, 2 \mathrm{H}, J=10.9 \mathrm{~Hz}$, $\left.\mathrm{H}_{\mathrm{Ar}}\right), 7.66$ and $7.68(\mathrm{~s}, 1 \mathrm{H}, \mathrm{s}, 1 \mathrm{H}, \mathrm{Ar}-\mathrm{CH}=\mathrm{C}$ and s, $1 \mathrm{H}, \mathrm{C}=\mathrm{CH}-\mathrm{Ar}) .{ }^{13} \mathrm{C}-\mathrm{NMR}\left(100 \mathrm{MHz}, \mathrm{CD}_{3} \mathrm{OD}\right)$

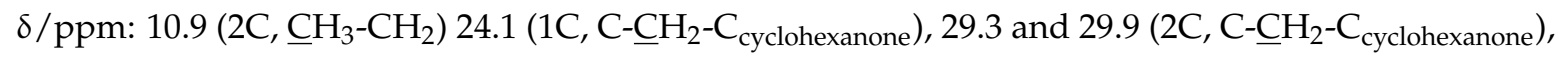

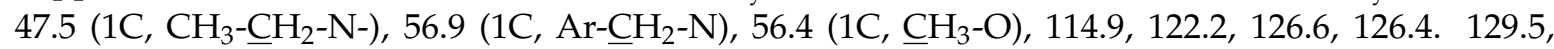
129.7, 131.3, 133.9 (10C, $\left.\mathrm{C}_{\mathrm{Ar}}\right), 137.2,137.3,137.9,139.7$ (4C, $\left.-\mathrm{C}=\mathrm{C}-\right), 149,7$ and $153.0\left(2 \mathrm{C}, \mathrm{C}_{\mathrm{Ar}} \mathrm{O}\right.$ ) $), 191.8$ $(1 \mathrm{C}, \mathrm{C}=\mathrm{O})$ [20] HRESIMS $(\mathrm{m} / \mathrm{z})$ found $404.2285\left([\mathrm{M}-\mathrm{H}]^{-}\right)$calculated masses for $\mathrm{C}_{26} \mathrm{H}_{30} \mathrm{NO}_{3}: 404.2226$.

(2E,6E)-2-(\{3-[(diethylamino)methyl]-4-hydroxy-5-methoxyphenyl\}methylidene)-6-[(4-methoxyphenyl)methylidene] cyclohexan-1-one (2b). The compound was an orange sticky powder, in a 46.8\% yield, m.p.: 98-99 ${ }^{\circ} \mathrm{C}$ and $\mathrm{Rf}=0.51$ (ethyl acetate: ethanol =1:1). FTIR $(\mathrm{KBr})$ vmax $\mathrm{cm}^{-1}: 3059$ (CH aromatic), 2970 ( $\mathrm{CH}$ aliphatic), $1734(\mathrm{C}=\mathrm{O}), 1556(\mathrm{C}=\mathrm{C}), 1595$ and $1510\left(\mathrm{C}=\mathrm{C}\right.$ aromatic) $1271(\mathrm{C}-\mathrm{N}), 1155(\mathrm{C}-\mathrm{O}) .{ }^{1} \mathrm{H}$ NMR (500 MHz, CD $\mathrm{OD}), \delta /$ ppm: $1.17\left(\mathrm{t}, 6 \mathrm{H}, J=7.1, \mathrm{CH}_{3}-\mathrm{CH}_{2}-\right), 1.78\left(\mathrm{~m}, 2 \mathrm{H}, \mathrm{C}-\mathrm{CH}_{2}-\mathrm{C}_{\text {cyclohexanone }}\right.$ ); $2.77\left(\mathrm{q}, 4 \mathrm{H}, J=7.1 \mathrm{~Hz}, \mathrm{CH}_{3}-\mathrm{CH}_{2}-\mathrm{N}\right), 2.88$ and $2.92\left(\mathrm{t}, 2 \mathrm{H}, J=5.4 \mathrm{~Hz},=\mathrm{C}_{-} \mathrm{CH}_{2}-\mathrm{C}_{\text {cyclohexanone }}\right.$ and $\mathrm{t}, 2 \mathrm{H}$,

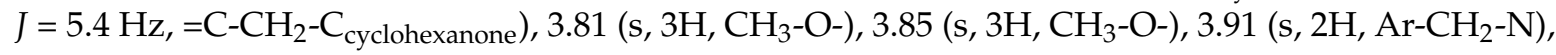
$6.89\left(\mathrm{~s}, 1 \mathrm{H}, \mathrm{H}_{\mathrm{Ar}}\right), 6.96\left(\mathrm{~d}, 2 \mathrm{H}, J=8.2, \mathrm{H}_{\mathrm{Ar}}\right), 7.01\left(\mathrm{~s}, 1 \mathrm{H}, \mathrm{H}_{\mathrm{Ar}}\right) ; 7.44\left(\mathrm{~d}, 2 \mathrm{H}, J=8.8 \mathrm{~Hz}, \mathrm{H}_{\mathrm{Ar}}\right), 7.64$ and 7.65

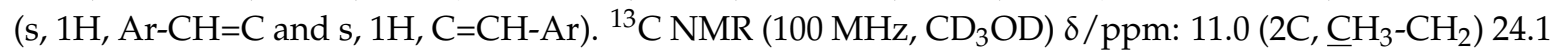
(1C, C- $\left.\mathrm{CH}_{2}-\mathrm{C}_{\text {cyclohexanone }}\right), 29.4$ and 29.6 (2C, C- $\left.\mathrm{CH}_{2}-\mathrm{C}_{\text {cyclohexanone }}\right), 47.4\left(2 \mathrm{C}, \mathrm{CH}_{3}-\underline{\mathrm{CH}}_{2}-\mathrm{N}-\right), 56.3(1 \mathrm{C}$, $\left.\mathrm{CH}_{3}-\mathrm{O}\right), 55.8\left(1 \mathrm{C}, \underline{\mathrm{CH}}_{3}-\mathrm{O}\right), 57.0\left(1 \mathrm{C}, \mathrm{Ar}-\underline{\mathrm{CH}}_{2}-\mathrm{N}\right), 114.8,122.8,126.7,126.2,126.7,133.4\left(9 \mathrm{C}, \mathrm{C}_{\mathrm{Ar}}\right), 129.7$, 135.6, 137.7, 139.3 (4C, $-\mathrm{C}=\mathrm{C}-$ ), 149.6, 152.8 and 161.7 (3C, $\left.\mathrm{C}_{\mathrm{Ar}}-\mathrm{O}\right), 191.9$ (1C, C=O) [20]. HRESIMS $(m / z)$ found $434.2101\left([\mathrm{M}-\mathrm{H}]^{-}\right)$calculated masses for $\mathrm{C}_{27} \mathrm{H}_{32} \mathrm{NO}_{4}: 434.2332$. 
(2E,6E)-2-(\{3-[(diethylamino)methyl]-4-hydroxy-5-methoxyphenyl\}methylidene)-6-[(4-fluorophenyl)methylidene] cyclohexan-1-one (2c). The compound was an orange powder, in a 33.01\% yield, m.p.: 79-81 ${ }^{\circ} \mathrm{C}$ and $\mathrm{Rf}=0.48$ (ethyl acetate: ethanol = 1:1). FTIR $(\mathrm{KBr})$ vmax $\mathrm{cm}^{-1}: 3041(\mathrm{CH}$ aromatic), 2937 (CH aliphatic), $1734(\mathrm{C}=\mathrm{O}), 1656(\mathrm{C}=\mathrm{C}), 1595$ and 1492 (C=C aromatic) 1271 (C-N),1224 (C-F), 1157 (C-O). ${ }^{1} \mathrm{H}-\mathrm{NMR}$ (500 MHz, CD $3 \mathrm{OD}), \delta / p p m: 1.18\left(\mathrm{t}, 6 \mathrm{H}, \mathrm{J}=7.2 \mathrm{~Hz}, \mathrm{CH}_{3}-\mathrm{CH}_{2}-\right), 1.77\left(\mathrm{~m}, 2 \mathrm{H}, \mathrm{C}-\mathrm{CH}_{2}-\mathrm{C}_{\text {cyclohexanone }}\right.$ ); $2.77\left(\mathrm{q}, 4 \mathrm{H}, \mathrm{J}=7.2 \mathrm{~Hz}, \mathrm{CH}_{3}-\mathrm{CH}_{2}-\mathrm{N}\right), 2.87$ and $2.94\left(\mathrm{t}, 2 \mathrm{H}, \mathrm{J}=5.5 \mathrm{~Hz},=\mathrm{C}-\mathrm{CH}_{2}-\mathrm{C}_{\text {cyclohexanone }}\right.$ and $\mathrm{t}$, $2 \mathrm{H}, J=5.2 \mathrm{~Hz}$, =C-CH $\left.-\mathrm{C}_{\text {cyclohexanone }}\right) ; 3.85$ (s, 3H, $\left.\mathrm{CH}_{3}-\mathrm{O}\right), 3.93\left(\mathrm{~s}, 2 \mathrm{H}, \mathrm{Ar}-\mathrm{CH}_{2}-\mathrm{N}\right), 6.92\left(\mathrm{~s}, 1 \mathrm{H}, \mathrm{H}_{\mathrm{Ar}}\right)$, $7.03\left(\mathrm{~s}, 1 \mathrm{H}, \mathrm{H}_{\mathrm{Ar}}\right) ; 7.14\left(\mathrm{~d}-\mathrm{d}, 2 \mathrm{H}, J=8.8 \mathrm{~Hz}, \mathrm{H}_{\mathrm{Ar}}\right), 7,49\left(\mathrm{~d}-\mathrm{d}, 2 \mathrm{H}, J=5.5 \mathrm{~Hz}, \mathrm{H}_{\mathrm{Ar}}\right), 7.65$ and $7.66(\mathrm{~s}, 1 \mathrm{H}$, $\mathrm{Ar}-\mathrm{CH}=\mathrm{C}$ and s, $1 \mathrm{H}, \mathrm{C}=\mathrm{CH}-\mathrm{Ar}) .{ }^{13} \mathrm{C}-\mathrm{NMR}\left(100 \mathrm{MHz}, \mathrm{CD}_{3} \mathrm{OD}\right) \delta / \mathrm{ppm}: 10.9\left(2 \mathrm{C}, \mathrm{CH}_{3}-\mathrm{CH}_{2}\right) 24.0$ (1C, C- $\left.-\mathrm{CH}_{2}-\mathrm{C}_{\text {cyclohexanone }}\right), 29.4$ and 29.6 (2C, C- $\left.\mathrm{CH}_{2}-\mathrm{C}_{\text {cyclohexanone }}\right), 47.4$ (2C, $\left.\mathrm{CH}_{3}-\mathrm{CH}_{2}-\mathrm{N}-\right), 56.3$ (1C, $\left.\mathrm{CH}_{3}-\mathrm{O}\right), 57.0$ (1C, $\left.\mathrm{Ar}-\underline{C H}_{2}-\mathrm{N}\right), 114.9,116.3,116.5,122.3,126.4,133.5,133.9\left(9 \mathrm{C}, \mathrm{C}_{\mathrm{Ar}}\right), 136.1,137.7,139.3$, 139.8 (4C, $-\mathrm{C}=\mathrm{C}-), 153.2$ and $163.0\left(2 \mathrm{C}, \mathrm{C}_{\mathrm{Ar}}-\mathrm{O}\right), 165.0\left(1 \mathrm{C}, \mathrm{C}_{\mathrm{Ar}}-\mathrm{F}\right), 191.6(1 \mathrm{C}, \mathrm{C}=\mathrm{O})$ [20]. HRESIMS $(\mathrm{m} / z)$ found $422.2178\left([\mathrm{M}-\mathrm{H}]^{-}\right)$calculated masses for $\mathrm{C}_{26} \mathrm{H}_{29} \mathrm{FNO}_{3}: 422.2132$.

(2E,6E)-2-(\{3-[(diethylamino)methyl]-4-hydroxy-5-methoxyphenyl\}methylidene)-6-[(4-chlorophenyl)methylidene] cyclohexan-1-one (2d). The compound was an orange powder, in a 76.93\% yield, m.p.: 95-97 ${ }^{\circ} \mathrm{C}$ and $\mathrm{Rf}=0.45$ (ethyl acetate: ethanol = 1:1). FTIR $(\mathrm{KBr})$ vmax $\mathrm{cm}^{-1}: 3032(\mathrm{CH}$ aromatic), $2972(\mathrm{CH}$ aliphatic), $1734(\mathrm{C}=\mathrm{O}), 1656(\mathrm{C}=\mathrm{C}), 1597$ and 1491 (C=C aromatic) $1271(\mathrm{C}-\mathrm{N}), 1157(\mathrm{C}-\mathrm{O}), 839(\mathrm{C}-\mathrm{Cl}) .{ }^{1} \mathrm{H}-\mathrm{NMR}$ (500 MHz, CD $3 \mathrm{OD}), \delta / p p m: 1.18\left(\mathrm{t}, 6 \mathrm{H}, \mathrm{J}=7.2 \mathrm{~Hz}, \mathrm{CH}_{3}-\mathrm{CH}_{2}-\right), 1.78\left(\mathrm{~m}, 2 \mathrm{H}, \mathrm{C}-\mathrm{CH}_{2}-\mathrm{C}_{\text {cyclohexanone }}\right)$; $2.79\left(\mathrm{q}, 4 \mathrm{H}, J=7.2 \mathrm{~Hz}, \mathrm{CH}_{3}-\mathrm{CH}_{2}-\mathrm{N}\right), 2.87$ and $2.95\left(\mathrm{t}, 2 \mathrm{H}, J=5.1 \mathrm{~Hz},=\mathrm{C}-\mathrm{CH}_{2}-\mathrm{C}_{\text {cyclohexanone }}\right.$ and $\mathrm{t}$, $\left.2 \mathrm{H}, \mathrm{J}=7.3 \mathrm{~Hz},=\mathrm{C}-\mathrm{CH}_{2}-\mathrm{C}_{\text {cyclohexanone }}\right) ; 3.85\left(\mathrm{~s}, 3 \mathrm{H}, \mathrm{CH}_{3}-\mathrm{O}\right), 3.94\left(\mathrm{~s}, 2 \mathrm{H}, \mathrm{Ar}-\mathrm{CH}_{2}-\mathrm{N}\right), 6.93\left(\mathrm{~s}, 1 \mathrm{H}, \mathrm{H}_{\mathrm{Ar}}\right)$, $7.04\left(\mathrm{~s}, 1 \mathrm{H}, \mathrm{H}_{\mathrm{Ar}}\right) ; 7.42\left(\mathrm{~d}, 2 \mathrm{H}, J=8.4 \mathrm{~Hz}, \mathrm{H}_{\mathrm{Ar}}\right), 7.44\left(\mathrm{~d}, 2 \mathrm{H}, J=8.7 \mathrm{~Hz}, \mathrm{H}_{\mathrm{Ar}}\right), 7.63$ and $7.66(\mathrm{~s}, 1 \mathrm{H}$, $\mathrm{Ar}-\mathrm{CH}=\mathrm{C}$ and $\mathrm{s}, 1 \mathrm{H}, \mathrm{C}=\mathrm{CH}-\mathrm{Ar}) .{ }^{13} \mathrm{C}-\mathrm{NMR}\left(100 \mathrm{MHz}, \mathrm{CD}_{3} \mathrm{OD}\right) \delta / \mathrm{ppm}: 11.0\left(2 \mathrm{C}, \mathrm{CH}_{3}-\mathrm{CH}_{2}\right), 24.1$ (1C, C- $\underline{C H}_{2}-\mathrm{C}_{\text {cyclohexanone }}$ ), 29.2 and 29.6 (2C, C- $\left.\underline{C H}_{2}-\mathrm{C}_{\text {cyclohexanone }}\right), 47.4\left(2 \mathrm{C}, \mathrm{CH}_{3}-\underline{\mathrm{CH}}_{2}-\mathrm{N}\right), 56.3$ (1C, $\left.\mathrm{CH}_{3}-\mathrm{O}\right), 56.9\left(1 \mathrm{C}, \mathrm{Ar}-\underline{C H}_{2}-\mathrm{N}\right), 114.9,122.5,126.5,129.6,133.7,132.8\left(9 \mathrm{C}, \mathrm{C}_{\mathrm{Ar}}\right), 136.1,137.7,138.5,139.8$ (4C, $-\mathrm{C}=\mathrm{C}-), 153.2$ and $149.7\left(2 \mathrm{C}, \mathrm{C}_{\mathrm{Ar}}-\mathrm{O}\right), 139.9\left(1 \mathrm{C}, \mathrm{C}_{\mathrm{Ar}}-\mathrm{Cl}\right), 191.6(1 \mathrm{C}, \mathrm{C}=\mathrm{O})$ [20]. HRESIMS $(\mathrm{m} / z)$ found $438.1881\left([\mathrm{M}-\mathrm{H}]^{-}\right)$calculated masses for $\mathrm{C}_{26} \mathrm{H}_{29} \mathrm{ClNO}_{3}: 438.1837$.

(2E,6E)-2-(\{3-[(diethylamino)methyl]-4-hydroxy-5-methoxyphenyl\}methylidene)-6-[(4-methylphenyl)methylidene] cyclohexan-1-one (2e). The compound was an orange sticky powder, in a 76.9\% yield, m.p.: 86-89 ${ }^{\circ} \mathrm{C}$ and $\mathrm{Rf}=0.48$ (ethyl acetate: ethanol =1:1). FTIR $(\mathrm{KBr})$ vmax $\mathrm{cm}^{-1}: 3032(\mathrm{CH}$ aromatic), $2974(\mathrm{CH}$ aliphatic), $1734(\mathrm{C}=\mathrm{O}), 1664$ (C=C), 1599 and 1498 (C=C aromatic) 1269 (C-N), 1157 (C-O). ${ }^{1} \mathrm{H}-\mathrm{NMR}(500 \mathrm{MHz}$,

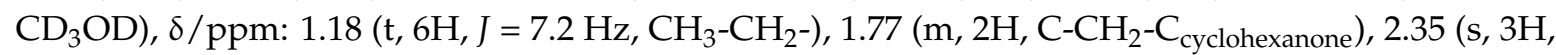
$\left.\mathrm{CH}_{3}-\mathrm{Ar}\right), 2.80\left(\mathrm{q}, 4 \mathrm{H}, J=7.2 \mathrm{~Hz}, \mathrm{CH}_{3}-\mathrm{CH}_{2}-\mathrm{N}\right), 2.90$ and $2.94\left(\mathrm{t}, 2 \mathrm{H}, J=7.3 \mathrm{~Hz},=\mathrm{C}-\mathrm{CH}_{2}-\mathrm{C}_{\text {cyclohexanone }}\right.$ and $\mathrm{t}, 2 \mathrm{H}, \mathrm{J}=5.4 \mathrm{~Hz},=\mathrm{C}-\mathrm{CH}_{2}-\mathrm{C}_{\text {cyclohexanone }}$ ); 3.85 (s, 3H, $\left.\mathrm{CH}_{3}-\mathrm{O}\right), 3.94\left(\mathrm{~s}, 2 \mathrm{H}, \mathrm{Ar}-\mathrm{CH}_{2}-\mathrm{N}\right), 6.92$ (s, $\left.1 \mathrm{H}, \mathrm{H}_{\mathrm{Ar}}\right), 7.04\left(\mathrm{~s}, 1 \mathrm{H}, \mathrm{H}_{\mathrm{Ar}}\right) ; 7.23\left(\mathrm{~d}, 2 \mathrm{H}, J=7.8 \mathrm{~Hz}, \mathrm{H}_{\mathrm{Ar}}\right), 7.37\left(\mathrm{~d}, 2 \mathrm{H}, J=7,9 \mathrm{~Hz}, \mathrm{H}_{\mathrm{Ar}}\right), 7.65$ and 7.67 (s, $1 \mathrm{H}, \mathrm{Ar}-\mathrm{CH}=\mathrm{C}$ and s, $1 \mathrm{H}, \mathrm{C}=\mathrm{CH}-\mathrm{Ar}) .{ }^{13} \mathrm{C}-\mathrm{NMR}\left(100 \mathrm{MHz}, \mathrm{CD}_{3} \mathrm{OD}\right) \delta / \mathrm{ppm}: 10.9\left(2 \mathrm{C}, \underline{\mathrm{CH}}_{3}-\mathrm{CH}_{2}\right)$, 21.4 (1C, $\left.\underline{\mathrm{CH}}_{3}-\mathrm{Ar}\right), 24.1$ (1C, C- $\underline{\mathrm{CH}}_{2}-\mathrm{C}_{\text {cyclohexanone }),} 29.3$ and 29.6 (2C, C- $\left.\underline{\mathrm{H}}_{2}-\mathrm{C}_{\text {cyclohexanone }}\right), 47.5$ (2C, $\left.\mathrm{CH}_{3}-\underline{C H}_{2}-\mathrm{N}\right), 54$ (1C, $\left.\underline{\mathrm{CH}}_{3}-\mathrm{O}\right), 56.9$ (1C, Ar- $\left.\underline{C H}_{2}-\mathrm{N}\right), 114.9,122.2,126.3,126.7,130.2,131.5,134.1$ (10C, $\left.\mathrm{C}_{\mathrm{Ar}}\right), 134.3,137.0,137.6,139.5$ (4C, $\left.-\mathrm{C}=\mathrm{C}-\right), 149.7$ and $152.9\left(2 \mathrm{C}, \mathrm{C}_{\mathrm{Ar}}-\mathrm{O}\right), 191.6(1 \mathrm{C}, \mathrm{C}=\mathrm{O})$ [20]. HRESIMS $(m / z)$ found $419.1925\left([\mathrm{M}-\mathrm{H}]^{-}\right)$calculated masses for $\mathrm{C}_{27} \mathrm{H}_{32} \mathrm{NO}_{3}: 419.2384$.

\subsection{Cytotoxicity Test}

\subsubsection{Brine Shrimp Lethality Test}

The assay was carried out according to the principle and protocol previously described by Meyer [23], with slight modification. Artemia salina L. eggs were inserted into a box containing seawater; the box was placed under UV lamp, and after $48 \mathrm{~h}$ the eggs hatched into larvae and ready for the test. The compounds (1a-1e, 2a-2e) were diluted in $10 \mathrm{~mL}$ seawater containing 10 larvae $(1 \%$ DMSO $(v / v))$ until concentrations 20, 200, 500, and 1000 ppm were reached. After $24 \mathrm{~h}$, the live 
and dead shrimp were counted. The mortality rate (\%) was obtained by comparing the number of total dead larvae and the total number of larvae. The experiment was conducted in triplicate. The concentrations (dose)-response (\% mortality) data were transformed into a straight line using a logit transformation, and the concentration required to kill $50 \%$ of the population ( $\mathrm{LC}_{50}$ values) was derived from the best fit line obtained by linear regression analysis.

\subsubsection{MTT Proliferation Assay}

The cytotoxic activity of the synthesized compounds was evaluated against human cervix carcinoma (HeLa, ATCC CCL-2) cell lines, human estrogen-dependent breast carcinoma (MCF7, ATCC HTB-22) cell lines, and kidney of an African green monkey (Vero, ATCC CCL-81) as a normal cell lines using the methyl thiazolyl tetrazolium (MTT) method conducted according to the MTT assay protocol published by the American Type Culture Collection (ATCC) [24]. Cisplatin was used as a reference drug. Curcumin was also evaluated as a comparative compound on the tests against Vero and MCF7 cell lines, and doxorubicin was evaluated as an additional comparator on the test against MCF7 cell lines. The assay detects the reduction of yellow tetrazolium (MTT) by metabolically active cells to be purple formazan measured using spectrophotometry [24].

The cells lines were seeded into 96-well plates at a density of 5000 cells per well, replenished with growth media consisting of Dulbecco's Modified Eagle's medium (D-MEM) for Vero or Roswell Park Memorial Institute (RPMI) 1640 medium for MCF7 and HeLa, 5\% Fetal Bovine Serum (FBS), $100 \mathrm{U} / \mathrm{mL}$ penicillin, and $100 \mu \mathrm{g} / \mathrm{mL}$ streptomycin. The cells were incubated at $37{ }^{\circ} \mathrm{C}$ in $5 \% \mathrm{CO}_{2}$ for $24 \mathrm{~h}$. Then, a series concentrations of the tested compounds (1.5, 3.1, 6.2, 12.5, 25.0, 50.0, 100.0, and $200.0 \mu \mathrm{g} / \mathrm{mL}$ ) were added to each well of the plate and incubated for $48 \mathrm{~h}$. After that, $10 \mu \mathrm{L}$ fresh solution of MTT reagent was added to each well, and the plate was incubated in a $\mathrm{CO}_{2}$ incubator at $37{ }^{\circ} \mathrm{C}$ for $4 \mathrm{~h}$. After the purple precipitate was obtained, the cells were dissolved in ethanol and their optical density was recorded at $595 \mathrm{~nm}$. The experiment was performed in triplicate. Percent proliferation inhibition was calculated using the following formula:

$$
\text { Viability cells inhibition }(\%)=100-\left[\frac{(\mathrm{At}-\mathrm{Ab})}{(\mathrm{Ac}-\mathrm{Ab})}\right] \times 100 \%
$$

At $=$ absorption of test compound, $\mathrm{Ab}=$ absorption of blank, $\mathrm{Ac}=$ absorption of control.

The concentration of the synthesized compounds required to inhibit $50 \%$ of the growth of the cell lines ( $\mathrm{IC}_{50}$ values) was calculated by analyzing the relationship between concentrations and percent (\%) inhibitions using GraphPad Prism 7 version 7.00 for Windows, GraphPad Software, La Jolla, CA, USA, www.graphpad.com [25].

\section{Results and Discussion}

\subsection{Chemistry}

The novel asymmetrical mono-carbonyl analogs of curcumin (AMACs) (1a-1e) and its diethylamine Mannich base derivatives (2a-2e) compounds were synthesized to further explore as anticancer compounds. The synthetic route of the compounds is shown in Scheme 1. The intermediate compounds, (2E)-2-(phenylmethylidene)cyclohexan-1-one and its analogs, were synthesized by the Claisen-Schmidt reaction between benzaldehyde or its analogs with cyclohexanone in the presence of aqueous alkali according to the preparation method of 4-phenylbut-3-en-2-one [19]. The aldol condensation of the intermediate compounds was obtained with vanilin with the addition of diluted $\mathrm{HCl}$ /ethanol under reflux conditions for $30 \mathrm{~min}$ gave AMACs (1a-1e). Finally, the Mannich reaction of 1a-1e with diethylamine and formaldehyde under reflux condition in ethanol for 7-11 h (TLC monitoring) afforded the diethylamine Mannich bases of AMACs compounds (2a-2e).

The FTIR spectra of compounds 1a-1e showed absorption bands at $3200-3500 \mathrm{~cm}^{-1}$ due to the presence of the $\mathrm{OH}$ group. The bands at about $1100 \mathrm{~cm}^{-1}$ correspond to $\mathrm{C}-\mathrm{O}-\mathrm{C}$ ether, whereas the 
$\alpha, \beta$-unsaturated carbonyl groups of the AMACs were observed as strong bands at about $1600 \mathrm{~cm}^{-1}$. In the ${ }^{1} \mathrm{H}-\mathrm{NMR}$ spectra, protons of $\mathrm{OH}$ phenolic and $\mathrm{OCH}_{3}$ group appear as a singlet at $\delta 5.86-5.90 \mathrm{ppm}$ $(1 \mathrm{H})$ and as a singlet at $\delta 3.91-3.93 \mathrm{ppm}(3 \mathrm{H})$, respectively. The two protons of ethenyl chain of the compounds appeared as two singlets at 7.72-7.79 ppm (2H), indicating an asymmetrical compound. The FTIR spectra of compounds $\mathbf{2 a} \mathbf{a}-\mathbf{2}$ showed the disappearance of $\mathrm{OH}$ phenolic. The bands at $1151-1271 \mathrm{~cm}^{-1}$ correspond to C-O-C and C-N, whereas the $\alpha, \beta$-unsaturated carbonyl groups of the compounds were observed as strong bands at $1,734 \mathrm{~cm}^{-1}$. In the ${ }^{1} \mathrm{H}-\mathrm{NMR}$ spectra, protons of the $\mathrm{OCH}_{3}$ group appeared as a singlet at $\delta 3.85 \mathrm{ppm}(3 \mathrm{H})$. The two protons of the ethenyl chain of the compounds were observed as two singlets in the range of 7.63 to $7.71 \mathrm{ppm}(1 \mathrm{H}$, respectively). The protons of diethylamine groups were observed at 1.16-1.18 as a triplet $(6 \mathrm{H})$ and $2.75-2.80 \mathrm{ppm}$ as a quintet $(4 \mathrm{H})$, and the protons of methylene adjacent $\mathrm{N}$ to the phenyl ring were observed as a singlet $(2 \mathrm{H})$ at 3.90-3.95 ppm. The proton signal of $\mathrm{OH}$ phenolic disappeared because of exchangeable with deuterium from $\mathrm{CD}_{3} \mathrm{OD}$ used as a solvent in the experiment [20]. Furthermore, the structures of the compounds were supported by ${ }^{13} \mathrm{C}-\mathrm{NMR}$ and HR-MS, which showed complete agreement with the expected molecular structures.
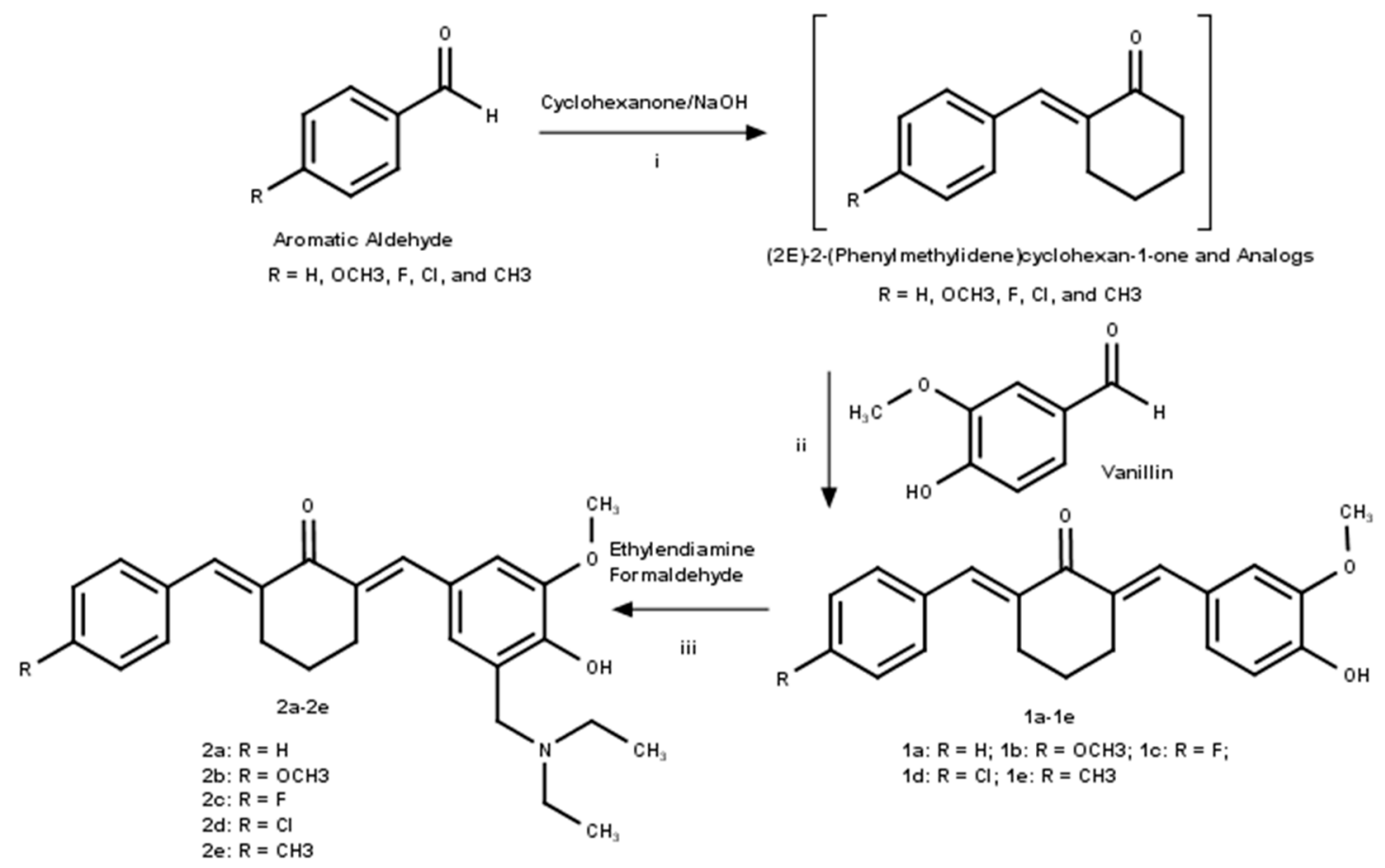

Scheme 1. Synthesis of the title compounds (1a-1e and 2a-2e). Conditions: (i) r.t., 2 h.; (ii) ethanol, reflux, diluted $\mathrm{HCl} /$ ethanol, $30 \mathrm{~min}$.; and (iii) ethanol, reflux, 7-11 h.

\subsection{Cytotoxic Activity}

The cytotoxic activity of the compounds was evaluated firstly using a brine shrimp lethality test (BSLT) method as a preliminary test. All the synthesized compounds exhibited toxic activity with $\mathrm{LC}_{50}$ value in the range of 29.80-1704.23 $\mu \mathrm{M}(13.06-714.49 \mu \mathrm{g} / \mathrm{mL})$ (Table 1). Compounds $\mathbf{1} \mathbf{b}, \mathbf{2 a}$, and $\mathbf{2 e}$ had moderate toxicity ( $\mathrm{LC}_{50}$ value $>100-1000 \mu \mathrm{g} / \mathrm{mL}$ ), compound $\mathbf{1 a}, \mathbf{1 c}, \mathbf{1 d}, \mathbf{2 b}$ and $\mathbf{2 c}$ had high toxic activity $\left(\mathrm{LC}_{50}>30-100 \mu \mathrm{g} / \mathrm{mL}\right.$ ), and $\mathbf{1 e}$ and $\mathbf{2 d}$ had very high toxic activity $\left(\mathrm{LC}_{50}<30 \mu \mathrm{g} / \mathrm{mL}\right)[26,27]$. The BSLT is a rapid, inexpensive, and simple method used to predict the toxicity level of the compounds. The method is not specific to antitumor activity. However, a positive correlation was found between BSLT toxicity and cytotoxicity toward some cell lines [27]. Therefore, in the present study, all the synthesized compounds were followed up to be evaluated for their potential as an anti-cancer agent. 
The cytotoxic activity of the compounds was evaluated against HeLa and Vero cell lines and for certain selected compounds against MCF7 cell lines. The $\mathrm{IC}_{50}$ values and selectivity index (SI) that obtained from the MTT assay are presented in Table 1 and Figure 1. Most of the synthesized compounds (1b-1e and $\mathbf{2 a}-\mathbf{2 e}$ ) exhibited cytotoxic activity against HeLa cell lines with $\mathrm{IC}_{50}$ value in the range of 40.65-95.55 $\mu \mathrm{M}$. In our experiment, cisplatin exhibited an $\mathrm{IC}_{50}$ value of $67.59 \mu \mathrm{M}$, but an earlier study [28] reported a much lower value $(12.3 \mu \mathrm{M})$. The reason for this difference could be due to differences in the conditions of the assay [26]. Based on the experimental data, the cytotoxicity of the compound was comparable with that of cisplatin. Unfortunately, all the synthesized compounds exhibited higher cytotoxic activity on Vero cell lines compared to HeLa cell lines (IC 50 value 3.94-16.15 $\mu \mathrm{M})$. As a result, the SI of the synthesized compounds was less than 1 , indicating that the synthesized compounds were more toxic to a normal cell than to cervix carcinoma cells. Their SI values were lower than that of cisplatin, which had an SI value of 1.26. The results also showed that most of the diethylamine Mannich base derivatives of AMACs (2a-2e) exhibited slightly higher cytotoxic activity against Hela cell lines than that of the parent compounds (1a-1e). This result is in line with a previous study that showed that the introduction of Mannich bases enhancing the biological activity of the compounds [29-33]. However, the increasing cytotoxicity was not selective because the effect was also observed at Vero cell lines.

The $\mathrm{IC}_{50}$ values of compounds $\mathbf{2 a}-\mathbf{2 d}$ to Vero cell lines were in the range of 3.94-7.28 $\mu \mathrm{M}$ $\left(1.73-3.17 \mu \mathrm{g} / \mathrm{mL}\right.$ ). Based on the cytotoxicity criteria of a pure compound ( $\mathrm{IC}_{50}<4 \mu \mathrm{g} / \mathrm{mL}$ or $<10 \mu \mathrm{M})[34,35]$, the compounds were considered highly toxic to the normal cell. Therefore, the cytotoxicity of $\mathbf{2 a - 2} \mathbf{d}$ against MCF7 cell lines was not evaluated. The results of the MTT assay against MCF7 cell lines showed that compound 1a exhibited noncytotoxic activity $\left(\mathrm{IC}_{50}>100 \mu \mathrm{M}\right)$, whereas compounds $\mathbf{1 b}-\mathbf{1 e}$ and $\mathbf{2 e}$ exhibited cytotoxic activity with $\mathrm{IC}_{50}$ values in the range of 7.86-35.88 $\mu \mathrm{M}$. Based on the data obtained, the cytotoxic activity of the synthesized compound is more selective to MCF7 cells rather than to HeLa cell lines. Among the synthesized compounds evaluated, compound $\mathbf{1 b}$ was the highest cytotoxic and selective compound against MCF7 cell lines with an $\mathrm{IC}_{50}$ value of $7.86 \mu \mathrm{M}(2.75 \mu \mathrm{g} / \mathrm{mL})$ and an SI value of 1.96 . The compound exhibited slightly higher cytotoxic activity $\left(\mathrm{IC}_{50}\right.$ value $\left.7.86 \mu \mathrm{M}\right)$ than that of curcumin and cisplatin $\left(\mathrm{IC}_{50}\right.$ values of 10.47 and $12.85 \mu \mathrm{M}$, respectively). However, the cytotoxicity was very low when compared with doxorubicin, which exhibits an $\mathrm{IC}_{50}$ value $<2.94 \mu \mathrm{M}$ (Table 1, Figure 1). Moreover, the selectivity index (SI) of the compounds to Vero and MCF7 was lower than that of curcumin and cisplatin with SI values of 1.96, 3.00, and 6.61, respectively (Table 1). The greater the SI value, the safer the compound.

The cytotoxicity of compound $\mathbf{1 b}$ containing a $4-\mathrm{OCH}_{3}$ group at the phenyl ring A (Table 1) was higher than $\mathbf{1 a}, \mathbf{1 d}, \mathbf{1 c}$, and 1e containing 4- $\mathrm{H}, 4-\mathrm{Cl}, 4-\mathrm{F}$, and $4-\mathrm{CH}_{3}$, respectively. The results were in line with earlier reported findings that substituent on 4-position of the phenyl ring of the AMACs or MACs significantly influenced the cytotoxicity of the compounds. In addition, a weak electron-donating substitution in 4-position was reported to be the most favorable to the cytotoxic activity of a compound [12,18]. Our data indicate that the electron-withdrawing substitution at 4-position reduced the cytotoxic activity. The effect differs from the electron-withdrawing substitution at 2-position which enhances the cytotoxic activity [12].

As per a standard used earlier, pure compounds are further considered for evaluation as chemotherapeutic agents in preclinical studies using an animal model, should have a potency of $10 \mu \mathrm{M}(4 \mu \mathrm{g} / \mathrm{mL})$ or less in cell culture studies and an SI value less than 2 [26,35]. Compound $\mathbf{1 b}$ could be considered as a new lead compound for further development to obtain more active and selective chemotherapeutic agents against breast cancer. 
Table 1. Cytotoxicity of the synthesized compounds against brine shrimp and Vero, Hela, and MCF7 cell lines.<smiles>[R]c1ccc(/C=C2\CCC/C(=C\c3cc([R])c(O)c(OC)c3)C2=O)cc1</smiles>

\begin{tabular}{|c|c|c|c|c|c|c|c|c|c|}
\hline \multirow{2}{*}{ No } & \multirow{2}{*}{ Compd } & \multicolumn{2}{|c|}{ Substuents } & \multirow{2}{*}{$\begin{array}{c}\mathrm{LC}_{50}(\mu \mathrm{M}) \\
\mathrm{BSLT}\end{array}$} & \multicolumn{3}{|c|}{$\mathrm{IC}_{50}(\mu \mathrm{M})^{(1)}$} & \multicolumn{2}{|c|}{ SI ${ }^{(2)}$} \\
\hline & & $\mathbf{R}_{1}$ & $\mathbf{R}_{2}$ & & Vero & Hela & MCF7 & Hela & MCF7 \\
\hline 1 & 1a & $\mathrm{H}$ & $\mathrm{H}$ & 196.63 & $13.98 \pm 0.04$ & $>100$ & $>100$ & $<1$ & $<1$ \\
\hline 2 & $1 b$ & $\mathrm{OCH}_{3}$ & $\mathrm{H}$ & 322.63 & $15.43 \pm 0.34$ & $95.55 \pm 7.19$ & $7.86 \pm 1.05$ & $<1$ & 1.96 \\
\hline 3 & 1c & $\mathrm{F}$ & $\mathrm{H}$ & 177.36 & $13.39 \pm 0.39$ & $49.15 \pm 1.17$ & $10.94 \pm 0.79$ & $<1$ & 1.28 \\
\hline 4 & 1d & $\mathrm{Cl}$ & $\mathrm{H}$ & 204.09 & $14.06 \pm 0.18$ & $55.60 \pm 1.49$ & $35.88 \pm 4.57$ & $<1$ & $<1$ \\
\hline 5 & 1e & $\mathrm{CH}_{3}$ & $\mathrm{H}$ & 78.71 & $16.15 \pm 0.18$ & $61.19 \pm 2.86$ & $10.39 \pm 0.36$ & $<1$ & 1.55 \\
\hline 6 & $2 a$ & $\mathrm{H}$ & $x$ & 921.08 & $4.14 \pm 0.21$ & $46.61 \pm 1.54$ & nt & $<1$ & - \\
\hline 7 & $2 b$ & $\mathrm{OCH}_{3}$ & $x$ & 80.21 & $7.29 \pm 0.12$ & $69.29 \pm 3.17$ & nt & $<1$ & - \\
\hline 8 & $2 c$ & $\mathrm{~F}$ & $x$ & 88.37 & $4.23 \pm 0.32$ & $41.10 \pm 0.16$ & nt & $<1$ & - \\
\hline 9 & $2 d$ & $\mathrm{Cl}$ & $x$ & 29.80 & $3.94 \pm 0.07$ & $40.65 \pm 0.98$ & nt & $<1$ & - \\
\hline 10 & $2 e$ & $\mathrm{CH}_{3}$ & $x$ & 1704.23 & $15.02 \pm 0.14$ & $76.61 \pm 4.27$ & $14.55 \pm 1.96$ & $<1$ & 1.03 \\
\hline 11 & Curcumin & - & - & $\mathrm{nt}$ & $31.41 \pm 0.41$ & nt & $10.47 \pm 1.10$ & - & 3.00 \\
\hline 12 & Cisplatin & - & - & nt & $84.66 \pm 2.09$ & $67.59 \pm 2.04$ & $12.85 \pm 1.35$ & 1.26 & 6.61 \\
\hline 13 & Doxorubici & in - & - & nt & nt & nt & $<2.94$ & - & - \\
\hline
\end{tabular}

(1) Values are the mean $\pm \mathrm{SD}(n=3) ; \mathrm{nt}=$ not tested. ${ }^{(2)} \mathrm{SI}=$ Selectivity Index $=\mathrm{IC}_{50}$ value normal cell $/ \mathrm{IC}_{50}$ value cancer cell. $\mathrm{X}=\mathrm{CH}_{2}-\mathrm{N}\left(\mathrm{CH}_{2}-\mathrm{CH}_{3}\right)_{2}$.

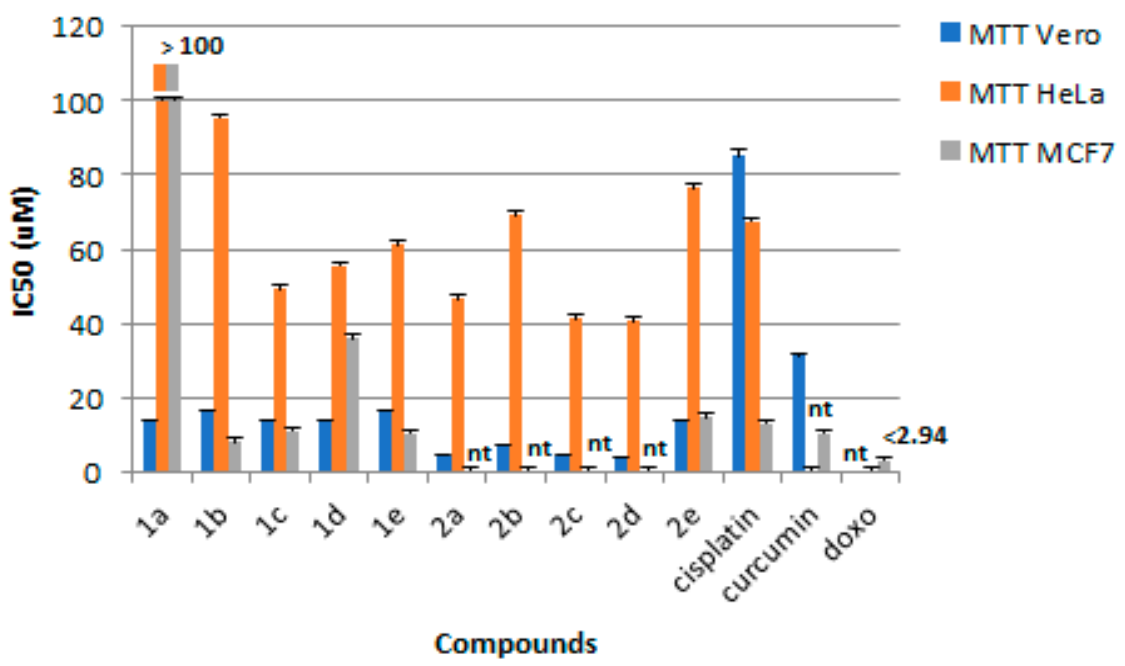

Figure 1. Cytotoxicity of the synthesized compounds against Vero, HeLa, and MCF7 Cell lines. $\mathrm{nt}=$ not tested.

Figure 2 depict the morphological analysis of untreated MCF7 cells (a) and Vero cell (b) versus treated MCF7 and Vero cell with respect to compound $\mathbf{1 b}(7.88 \mu \mathrm{M})(\mathrm{c}$ and d), curcumin $(8.48 \mu \mathrm{M})$ (e and $\mathrm{f})$, and cisplatin $(12.59 \mu \mathrm{M})(\mathrm{g}$ and $\mathrm{h})$. The figures compare the cytotoxicity of the compounds at the same concentration against human breast cancer cells MCF7 and normal cell Vero. 

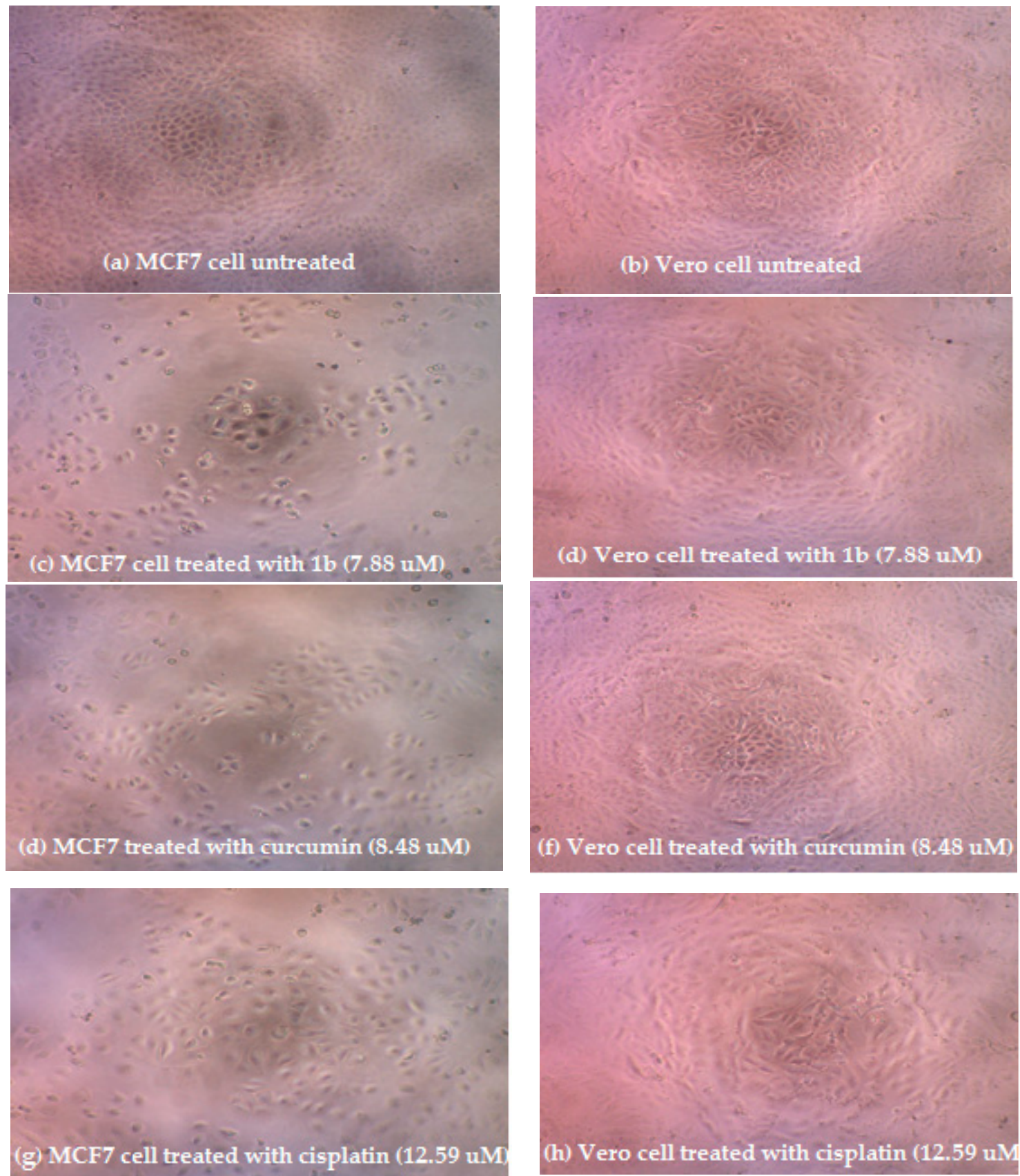

Figure 2. Morphological assessment of MCF7 cells (left) and Vero cells (right) using MTT assay. $(\mathbf{a}, \mathbf{b})$ untreated cells; $(\mathbf{c}, \mathbf{d})$ cells treated with compound $\mathbf{1 b}$ at $7.88 \mu \mathrm{M} ;(\mathbf{e}, \mathbf{f})$ cells treated with curcumin at $8.48 \mu \mathrm{M}$; and $(\mathbf{g}, \mathbf{h})$ cells treated with cisplatin at $12.59 \mu \mathrm{M}$.

\section{Conclusions}

A series of asymmetrical mono-carbonyl analogs of curcumin (AMACs) were successfully synthesized. All the synthesized compounds exhibited moderate to very high toxicity based on BSLT, most of them exhibited comparable cytotoxic activity with cisplatin against HeLa cell lines, and the selected compound exhibited moderate to very high cytotoxic activity against MCF7 cell lines. However, all compounds had low SI $(<1-1.96)$. Among the synthesized compounds, compound $\mathbf{1 b}$ showed the highest cytotoxic and selective activity against MCF7 cell lines. This compound could be considered for further development to obtain the more active and selective chemotherapeutic agents against breast cancer. 
Author Contributions: H.H. and A.B. conceived and designed the experiments; P.W.P. performed the experiments; H.H. and P.W.P. analyzed the data; and H.H. and P.W.P. wrote the paper.

Acknowledgments: We thank the Directorate of Research and Community Services, Directorate of Higher Education of the Ministry of Research, Technology, and Higher Education of the Republic of Indonesia for the financial support of this research (PDUPT Research Grant, 2017). We are also thankful to Chemistry Study Program, Faculty of Natural Sciences, Bandung Institute of Technology (ITB), Bandung, Indonesia for recording NMR spectral data; to Central Laboratory of Universitas Padjadjaran, Bandung, Indonesia for recording HR-MS data; to the Department of Chemistry, Faculty of Medicine, Universitas Indonesia, Jakarta, Indonesia and Institute for Research and Community Service, Primate Research Center, Bogor Agricultural University, Bogor, Indonesia, for MTT viability assay; and to Center for Chemistry Research, the Indonesian Institute of Sciences, Serpong, Tangerang, Indonesia for BSLT assay.

Conflicts of Interest: The authors declare no conflict of interest.

\section{References}

1. The World Health Organization. Cancer Country Profile, 2014. Available online: http://www.who.int/ cancer/country-profiles/idn_en.pdf?ua (accessed on 11 December 2017).

2. Roche, V.F. Cancer and chemotherapy. In Foye's Principles of Medicinal Chemistry, 11th ed.; Lemke, T.L., Williams, D.A., Roche, V.F., Zito, S.W., Eds.; Lippincott Wiliams and Wilkins: Baltimore, MD, USA, 2016; pp. 1199-1266.

3. Basnet, P.; Skalko-Basne, N. Curcumin: An Anti-Inflammatory Molecule from a Curry Spice on the Path to Cancer Treatment. Molecules 2011, 16, 4567-4598. [CrossRef] [PubMed]

4. Anand, P.; Thomas, S.G.; Kunnumakkara, A.B.; Sundaram, C.; Harikumar, K.B.; Sung, B.; Tharakan, S.T.; Misra, K.; Priyadarsini, I.K.; Rajasekharan, K.N.; et al. Biological activities of curcumin and its analogues (Congeners) made by man and Mother Nature. Biochem. Pharmacol. 2008, 76, 1590-1611. [CrossRef] [PubMed]

5. Anand, P.; Kunnumakkara, A.B.; Newman, R.A.; Aggarwal, B.B. Bioavalibility of curcumin: Problems and promises. Mol. Pharm. 2007, 4, 807-818. [CrossRef]

6. Sharma, R.A.; Euden, S.A.; Platton, S.L.; Cooke, D.N.; Shafayat, A.; Hewitt, H.R.; Marczylo, T.H.; Morgan, B.; Hemingway, D.; Plummer, S.M.; et al. Phase I clinical trial of oral curcumin: Biomarkers of systemic activity and compliance. Clin. Cancer Res. 2004, 10, 6847-6854. [CrossRef] [PubMed]

7. Garcea, G.; Berry, D.P.; Jones, D.J.; Singh, R.; Dennison, A.R.; Farmer, P.B.; Sharma, R.A.; Steward, W.P.; Gescher, A.J. Consumption of the putative chemopreventive agent curcumin by cancer patients: Assessment of curcumin levels in the colorectum and their pharmacodynamic consequences. Cancer Epidemiol. Biomark. Prev. 2005, 14, 120-125.

8. Shetty, D.; Kim, Y.J.; Shim, H.; Snyder, J.P. Eliminating the Heart from the Curcumin Molecule: Monocarbonyl Curcumin Mimics (MACs). Molecules 2015, 20, 249-292. [CrossRef] [PubMed]

9. Ohori, H.; Yamakoshi, H.; Tomizawa, M.; Shibuya, M.; Kakudo, Y.; Takahashi, A.; Takahashi, S.; Kato, S.; Suzuki, T.; Ishioka, C.; et al. Synthesis and biological analysis of new curcumin analogues bearing an enhanced potential for the medicinal treatment of cancer. Mol. Cancer Ther. 2006, 5, 2563-2571. [CrossRef] [PubMed]

10. Yin, S.; Zheng, X.; Yao, X.; Wang, Y.; Liao, D. Synthesis and Anticancer Activity of Mono-Carbonyl Analogues of Curcumin. J. Cancer Ther. 2013, 4, 113-123. [CrossRef]

11. Adams, B.K.; Ferstl, E.M.; Davis, M.C.; Herold, M.; Kurtkaya, S.; Camalier, R.F.; Hollingshead, M.G.; Kaur, G.; Sausville, E.A.; Rickles, F.R.; et al. Synthesis and biological evaluation of novel curcumin analogs as anti-cancer and anti-angiogenesis agents. Bioorg. Med. Chem. 2004, 12, 3871-3883. [CrossRef] [PubMed]

12. Liang, G.; Shao, L.; Wang, Y.; Zhao, C.; Chu, Y.; Xiao, J.; Zhao, Y.; Li, X.; Yang, S. Exploration and synthesis of curcumin analogues with improved structural stability both in vitro and in vivo as cytotoxic agents. Bioorg. Med. Chem. 2009, 17, 2623-2631. [CrossRef] [PubMed]

13. Zhao, C.; Liu, Z.; Liang, G. Promising curcumin-based drug design: Monocarbonyl analogues of curcumin (MACs). Curr. Pharm. Res. 2013, 19, 2114-2135. [CrossRef] 
14. Zhang, Y.; Zhao, L.; Wu, J.; Jiang, X.; Dong, L.; Xu, F.; Liang, G. Synthesis and evaluation of a series of novel asymmetrical curcumin analogs for the treatment of inflammation. Molecules 2014, 19, 7287-7307. [CrossRef] [PubMed]

15. Zhang, Y.; Jiang, X.; Peng, K.; Chen, C.; Fi, L.; Wang, Z.; Feng, J.; Liu, Z.; Zhang, H.; Liang, G.; et al. Discovery and evaluation of novel anti-inflammatory derivatives of natural bioactive curcumin. Drug Des. Dev. Ther. 2014, 8, 2161-2171. [CrossRef]

16. Aluwi, M.F.M.F.; Rullah, K.; Yamin, B.M.; Leong, S.W.; Bahari, M.N.A.; Lim, S.J.; Lam, K.W. Synthesis of unsymmetrical monocarbonyl curcumin analogues with potent inhibition on prostaglandin E2 production in LPS-induced murine and human macrophages cell lines. Bioorg. Med. Chem. Lett. 2016, 26, 2531-2538. [CrossRef] [PubMed]

17. Bandgar, B.P.; Kinkar, S.N.; Chavan, H.V.; Jalde, S.S.; Shaikh, R.U.; Gacche, R.N. Synthesis and biological evaluation of asymmetric indole curcumin analogs as potential anti-inflammatory and antioxidant agents. J. Enzym. Inhib. Med. Chem. 2014, 29, 7-11. [CrossRef] [PubMed]

18. Li, Q.; Chen, J.; Luo, S.; Xu, J.; Huang, Q.; Liu, T. Synthesis and assessment of the antioxidant and antitumor properties of asymmetric curcumin analogues. Eur. J. Med. Chem. 2015, 93, 461-469. [CrossRef] [PubMed]

19. Furniss, B.S.; Hannaford, A.J.; Smith, P.W.G.; Tatchell, A.R. Vogel's Textbook of Practical Organic Chemistry, 5th ed.; Longman Group Ltd.: London, UK, 1989; pp. 1032-1033.

20. Silverstein, R.M.; Webster, F.X.; Kiemle, D.J. Spectrometric Identification of Organic Compounds, 7th ed.; John Wiley \& Sons, Inc.: New York, NY, USA, 2005.

21. Geschickter, C.F.; Meadow, J.R. Curcumin Derivatives. U.S. Patent 3,479,345, 18 November 1969.

22. Untung, J.; Iskandarsyah, I.; Hayun, H. 2-[(2,6-Dimethylmorpholin-4-yl)methyl]-4-[(E)-2-\{3-[(E)-2-\{3-[(2,6dimethylmorpholin-4-yl)methyl]-4-hydroxy-5-methoxyphenyl\}ethenyl]-1H-pyrazol-5-yl\}ethenyl]-6methoxyphenol. Molbank 2017, 3, M949. [CrossRef]

23. Meyer, B.N.; Ferrigni, N.R.; Putnam, J.E.; Jacobson, L.B.; Nichols, D.E.; McLaughlin, J.L. Brine shrim.p.: A convenient general bioassay for active plant constituents. Planta Med. 1982, 45, 31-34. [CrossRef] [PubMed]

24. MTT Cell Proliferation Assay. Available online: https://www.atcc.org/ /media/ DA5285A1F52C414E864C966FD78C9A79.ashx (accessed on 5 October 2017).

25. GraphPad Software, Inc. Available online: www.graphpad.com (accessed on 13 December 2017).

26. Badisa, R.B.; Darling-Reed, S.F.; Joseph, P.; Cooperwood, J.S.; Latinwo, L.M.; Goodman, C.B. Selective Cytotoxic Activities of Two Novel synthetic Drugs on Hyman Breast Carcinoma MCF-7 Cells. Anticancer Res. 2009, 29, 2993-2996. [PubMed]

27. Chisalberti, E.L. Detection and Isolation of Bioactive Natural Products. In Bioactive Natural Products, Detection, Isolation, and Structur Determination, 2nd ed.; Colegate, S.M., Molyneux, R.J., Eds.; Taylor and Francis Groups, LLC: Boca Raton, FL, USA, 2008; p. 18.

28. Aydin, S.; Becit, M.; Basaran, A.; Basaran, N. Effects of curcumin on cisplatin cytotoxicity in HeLa cells. Toxicol. Lett. 2016, 258, S259-S260. [CrossRef]

29. Bala, S.; Sharma, N.; Kajal, A.; Kamboj, S.; Saini, V. Mannich Bases: An Important Pharmacophore in Present Scenario. Int. J. Med. Chem. 2014, 2014, 191072. [CrossRef] [PubMed]

30. Liu, C.; Dong, L.; Wang, S.; Wang, Q. Synthesis and antiproliferative activity of pterostilbene and $3^{\prime}$-methoxy pterostilbene Mannich base derivatives against Hela cells. Mol. Divers. 2015, 19, 737-743. [CrossRef] [PubMed]

31. Yerdelen, K.O.; Gul, H.I.; Sakagami, H.; Umemura, N. Synthesis and biological evaluation of 1,5-bis(4-hydroxy-3-methoxyphenyl)penta-1,4-dien-3-one and its aminomethyl derivatives. J. Enzym. Inhib. Med. Chem. 2015, 30, 383-388. [CrossRef] [PubMed]

32. Reddy, M.V.B.; Su, C.-R.; Chiou, W.-F.; Liu, Y.-N.; Chen, R.Y.; Bastow, K.F.; Lee, K.-H.; Wu, T.-S. Design, synthesis, and biological evaluation of Mannich bases of heterocyclic chalcone analogs as cytotoxic agents. Bioorg. Med. Chem. 2008, 16, 7358-7370. [CrossRef] [PubMed]

33. Roman, G. Mannich bases in medicinal chemistry and drug design. Eur. J. Med. Chem. 2015, 89, 743-816. [CrossRef] [PubMed] 
34. Schmitz, F.J.; Bourden, B.F.; Toth, S.I. Antitumor and Cytotoxic Compounds from Marine Organisms. In Marine Biotechnology, Pharmaceutical and Bioactive Natural Products; Attaway, D.H., Zaborsky, O.R., Eds.; Plenum Press: New York, NY, USA, 1993; Volume 1, p. 198.

35. Burger, A.M.; Fiebig, H.H. Preclinical Screening for New Anticancer Agents. In Handbook of Anticancer Pharmacokinetics and Pharmacodynamics, Cancer Drug Discovery and Development; Figg, W.D., McLeod, H.L., Eds.; Humana Press Inc.: Totowa, NJ, USA, 2004; pp. 36-37.

(c)

(C) 2018 by the authors. Licensee MDPI, Basel, Switzerland. This article is an open access article distributed under the terms and conditions of the Creative Commons Attribution (CC BY) license (http:/ / creativecommons.org/licenses/by/4.0/). 